\title{
Pretreatment inflammatory indices predict Bevacizumab response in recurrent Glioma
}

\author{
Alicia Martínez-González ${ }^{1}$, Raquel Cabrera², Marta Lloret ${ }^{2,3,4}$, Pedro C. Lara ${ }^{4,5,6}$ \\ 'Department de Matemáticas, Universidad de Castilla-La Mancha, ETSI Industriales, Avda, Ciudad Real 13071, Spain. \\ 2Oncología Radioterápica Hospital Universitario de Gran Canaria Dr Negrin, Barranco de la Ballena s/n, Las Palmas de Gran \\ Canaria, Las Palmas 35010, Spain. \\ ${ }^{3}$ Facultad de Ciencias de la Salud, Universidad de Las Palmas de Gran Canaria, Paseo de Blas Cabrera Felipe, s/n, Las Palmas de \\ Gran Canaria, Las Palmas 35016, Spain. \\ ${ }^{4}$ Fundación Canaria del Instituto Canario de Investigación del Cáncer, Avda de la Trinidad 61 Torre Agustín Arevalo 7 planta La \\ Laguna, Santa Cruz de Tenerife 38204, Spain. \\ ${ }_{5}^{5}$ San Roque University Hospitals, Dolores de la Rocha, 5, Las Palmas de Gran Canaria, Las Palmas 35001, Spain. \\ ${ }_{6}^{6}$ Fernando Pessoa Canarias University, Dolores dela Rocha 14, Las Palmas de Gran Canaria, Las Palmas 35016, Spain.
}

Correspondence to: Dr. Alicia Martínez-González, Department of Mathematics, Mathematical Oncology Laboratory, Universidad de Castilla La Mancha, ETSI Industriales Avda. Camilo José Cela 3, Ciudad Real 13071, Spain. E-mail: Alicia.martinez@uclm.es

How to cite this article: Martínez-González A, Cabrera R, Lloret M, Lara PC. Pretreatment inflammatory indices predict Bevacizumab response in recurrent Glioma. Cancer Drug Resist2020;3:623-35. http://dx.doi.org/10.20517/cdr.2020.33

Received: 30 Apr 2020 First Decision: 7 May 2020 Revised: 18 May 2020 Accepted: 7 Jun 2020 Available online: 7 Aug 2020

Academic Editor: Chiara Riganti Copy Editor: Xin-Qian Li Production Editor: Jing Yu

\begin{abstract}
Aim: It remains unclear what the best therapeutic option for recurrent glioma patients after Stupp treatment is. Bevacizumab (BVZ) is commonly administered in progression, but it appears that only some patients benefit. It would be useful to find biomarkers that determine beforehand who these patients are.
\end{abstract}

Methods: The protocol included 31 high-risk progressing glioma patients after Stupp treatment who received BVZ 5-10 mg/kg every 14 days and temozolomide (3-19 cycles, 150-200 mg five days each 28-day cycle) during a mean of eight cycles of BVZ or until tumor progression or unacceptable toxicity. We analyzed the clinical outcome values of inflammatory indices measured before BVZ administration.

Results: Lymphocyte level before BVZ administration was the best independent predictor of overall survival (HR = $0.34 ; 95 \% \mathrm{Cl}: 0.145-0.81 ; P=0.015)$. The area under the receiver operating characteristic (ROC) curve was 0.823 , with 1.645 being the optimal cut-off value, and 0.80 and 0.85 the sensitivity and specificity values, respectively. Responder and non-responder survival curves were also significantly different, considering the first and second tertiles as cut-off points. The number of BVZ cycles was not related to lymphopenia. Pretreatment neutrophil,

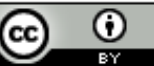

(C) The Author(s) 2020. Open Access This article is licensed under a Creative Commons Attribution 4.0 International License (https://creativecommons.org/licenses/by/4.0/), which permits unrestricted use, sharing, adaptation, distribution and reproduction in any medium or format, for any purpose, even commercially, as long as you give appropriate credit to the original author(s) and the source, provide a link to the Creative Commons license, and indicate if changes were made.

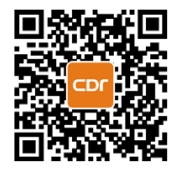


platelet levels, platelet-to-lymphocyte ratio (PLR), and neutrophil-to-lymphocyte ratio (NLR) did not have independent predictive value. Inflammatory variables were not correlated with each other. However, patients with high NLR and PLR simultaneously (double positive PLR-NLR) showed a worse clinical outcome than the rest $(P=$ 0.043).

Conclusion: Pretreatment lymphocyte levels and double positive PLR-NLR could be used as non-invasive hematological prognostic markers for recurrent gliomas treated with bevacizumab. A close relationship emerged between inflammation and angiogenesis.

Keywords: Recurrent glioma, bevacizumab, predictive biomarker, inflammatory indices, lymphocytes

\section{INTRODUCTION}

Primary brain tumors constitute about $2 \%$ of all malignant tumors; half of them are high-grade gliomas ${ }^{[1,2]}$ and some low-grade gliomas show aggressive transformation, becoming high-grade gliomas, including glioblastoma $(\mathrm{GBM})^{[3]}$. GBM aggressiveness leads to a median survival time of around three months without treatment, and 12-15 months with all therapeutic interventions ${ }^{[4]}$. Only $8 \%-12 \%$ of patients survive for two years and fewer than $3 \%$ of patients survive for five years ${ }^{[1,5-7]}$. Standard treatment includes maximal safe surgical resection and the Stupp protocol: concomitant temozolomide (TMZ) (75 mg seven days/week) to radiotherapy (RT) and adjuvant TMZ (six cycles, 150-200 mg five days each 28-day cycle). Progression and recurrence are observed with a median time of 10 weeks after finishing the standard treatment ${ }^{[1]}$. Tumor progression is associated with deterioration of neurocognitive function and a decrease in quality of life ${ }^{[5]}$. When progression occurs after the Stupp protocol, there is no standard treatment ${ }^{[8]}$. Therapeutic options then depend on tumor and patient characteristics: second surgery, re-irradiation, or chemotherapy such as TMZ, bevacizumab (BVZ), fotemustine, lomustine, cyclophosphamide, or irinotecan ${ }^{[9]}$. GBM is characterized by increased expression of vascular endothelial growth factor (VEGF), which stimulates angiogenesis ${ }^{[8,10-12]}$. BVZ is a humanized monoclonal immunoglobulin G1 which binds to VEGF. BVZ may reduce neo-vasculature, improve blood vessel integrity, and increase the percentage of apoptotic cells ${ }^{[9,13]}$. Since BVZ can only cross the blood-brain barrier in disrupted areas ${ }^{[14]}$, it is associated with non-enhancing tumor progression, improvement of clinical performance, and progression-free survival ${ }^{[15,16]}$. BVZ remains one of the few Food and Drug Administration (FDA) approved and most commonly prescribed therapies for recurrent GBM. However, the reduction in tumor size following therapy is transient and it is unclear which patients are most likely to benefit from treatment ${ }^{[17,18]}$.

GBM patient prognosis mainly depends on treatment administered, age, and Methyl Guanine Methyl Transferase (MGMT) methylation. Other markers seem to be related to survival: sex, Karnofsky Performance Status (KPS), Mini-Mental score, tumor location, necrosis, contrast-enhancement on preoperative Magnetic Resonance Image (MRI), or MRI texture variables ${ }^{[19-22]}$. A histopathological, molecular, immunohistochemical, and neuroradiological study did not find any predictive marker of response or survival benefit for BVZ in $\mathrm{GBM}^{[23]}$.

As generalized indicators of inflammation, the complete blood cell counts and their ratios have been investigated as biomarkers in several cancers and they have been found to be very accessible prognostic markers ${ }^{[24-29]}$. High lymphocytic infiltration is associated with improving survival and superior response to systemic therapy, whereas a low peripheral blood lymphocyte count is related to poor cancer prognoses ${ }^{[26]}$. Neutrophil infiltration stimulates tumor growth, angiogenesis, and metastasis through the secretion of VEGF, while platelets can also support cancer progression by promoting proliferation, angiogenesis and metastasis $^{[24-26]}$. Preoperative neutrophil-to-lymphocyte ratio (NLR) could reflect tumor burden and 
clinical outcomes in ovarian cancer, pancreatic cancer, renal cell carcinoma, and colorectal carcinoma. The prognostic value of NLR was the highest in mesothelioma and pancreatic cancer. Prognostic value of platelets-to-lymphocyte ratio (PLR) has been evaluated in colorectal, gastroesophageal, hepatocellular, pancreatic, and ovarian cancer ${ }^{[27-29]}$. NLR was significantly associated with improved results after addition of BVZ in a previous metastatic colorectal study.

However, we know less about the roles that inflammatory indices play as biomarkers for GBM patients ${ }^{[30-33]}$. NLR showed prognostic impact for GBM to first progression in patients undergoing second surgery ${ }^{[30-32]}$. NLR and PLR are considered prognostic markers in new gliomas: the higher are the ratios, the worse is the tumor prognosis. Pretreatment NLR is of prognostic significance independent of MGMT status and is superior to PLR as a prognostic factor ${ }^{[33]}$. Some authors have suggested a decision-tree-based model for recurrent GBM but peripheral blood information and response to BVZ were not considered ${ }^{[34]}$.

There are few studies focused on prognostic inflammatory biomarkers for recurrent gliomas and even fewer focusing on BVZ response. Only patients with a high neutrophil count benefited from BVZ in an exploratory cohort of patients treated with radio-chemotherapy, and then BVZ or chemotherapy at recurrence. BVZ use increased median overall survival (OS) $(18.7 \text { months } v s .11 .3 \text { months, } P=0.0014)^{[35]}$. However, a recent study found that neutrophils did not have predictive value for recurrent gliomas treated with BVZ. This study suggested the use of blood count changes over the entire duration of BVZ therapy to predict response. Changes in lymphocyte counts predicted survival time from BVZ initiation to death ${ }^{[35,36]}$. Nevertheless, it would be desirable to find a predictive biomarker obtained before treatment begins. To our knowledge, no one has analyzed NLR and PLR with survival in recurrent gliomas treated with BVZ.

In this study, we analyzed serum levels before treatment with BVZ to determine whether lymphocytes, neutrophils, platelets, NLR, or PLR can be proposed as prognostic markers of overall survival in recurrent gliomas treated with BVZ. The final aim was to classify patients who receive more benefit from the treatment with BVZ, considering new immune markers.

\section{METHODS}

\section{Patients}

Data on 31 patients with recurrent glioma were retrospectively collected and analyzed. Clinical variables such as age, sex, preoperative KPS, extent of resection, localization, histopathology, and adjuvant therapy were included. After first surgery, most patients received radiotherapy (93.55\%) and concomitant temozolomide (80.64\%). Patients had follow-ups with serial MRI, so progression was defined as clinical progression, radiological progression, or both. Tumor progression was defined according to the modified World Health Organization (WHO) criteria as an appearance of new lesions, an increase in tumor size by $25 \%$ in radiological images, or an increased need for corticosteroids ${ }^{[32]}$. The median time from diagnosis of high-grade glioma to recurrence was 15 months. From progression diagnosis, six patients underwent surgery, five of whom were GBM at baseline and the other had a grade II astrocytoma that progressed to GBM. Patients who did not receive surgery were categorized as their previous histological subtype and only two patients were irradiated. This study was carried out in accordance with the protocol "PATRORA" approved by the Agencia Española de Medicamentos y Productos Sanitarios (Ref. PLJ-BEV-2016-01) and by the Hospital Universitario de Gran Canaria Doctor Negrín committee (Ref. 170007).

\section{Inflammatory data and toxicity}

The levels of neutrophils, platelets, and lymphocytes were measured. To study the association between inflammatory response and recurrent gliomas, median and tertile levels of neutrophils, lymphocytes, and platelets were used as cut-off values to discriminate between groups. 
NLR and PLR were calculated by dividing the absolute neutrophil or platelet counts, respectively, by the absolute lymphocyte count, using the hemogram prior to bevacizumab treatment. Median NLR and PLR values were used to classify patients into positive (above the median) or negative (below the median) NLR and PLR groups, respectively. Patients with positive NLR and PLR simultaneously were included in the double-positive group while those with negative NLR and PLR simultaneously were in the double-negative group. In any other case, the patient was considered as single positive.

\section{Statistical analysis}

Statistical analysis was performed using Statistical Package for The Social Science software (SPSS). For all analyses, a $P$-value $<0.05$ was accepted as significant. First, the Kolmogorov-Smirnov test was used to identify whether the variables followed a normal distribution to choose a parametric or non-parametric test. Comparisons among groups were performed using the $\chi^{2}$ test for categorical variables, and KruskalWallis and Mann-Whitney tests for continuous variables. $\chi^{2}$ and T-student tests were used to compare the distribution differences of each variable. Correlation between variables was performed using the Spearman test. Survival analysis was evaluated through Kaplan-Meier curves and comparison between subgroups was reproduced by a Log-Rank probability test. Cox proportional hazards regression analysis was used to obtain the $\mathrm{HR}$ and its adjusted $95 \% \mathrm{CI}$ for each threshold. The area under the receiver operating characteristic (ROC) curve was calculated to evaluate optimal cut-off values.

\section{RESULTS}

\section{Descriptive data analysis}

After Stupp treatment, all patients received BVZ 5-10 mg/kg every 14 days and $94 \%$ combined the BVZ with temozolomide (3-19 cycles, 150-200 mg five days each 28-day cycle) during a mean of eight cycles of BVZ or until tumor progression or unacceptable toxicity. Fifty-five percent received temozolomide again in accordance with the Stupp protocol, five days every 28 days at a dose of $150-200 \mathrm{mg} / \mathrm{m}^{2}$ and $38 \%$ a metronomic daily dose of 40-60 mg. Table 1 summarizes the main patient features included in the study. In fact, $55.17 \%$ of the patients received Stupp treatment after progression (4.5 average cycles); however, this did not affect later neutropenia.

At the end of the study, 26 patients had died (83.8\%), 2 continued receiving bevacizumab cycles as they were in a state of remission, and 1 patient showed a response above $50 \%$. The median survival from BVZ administration was 6.5 months (95\%CI: 4.1-8.9 months) and long-term survivals represented 9.6\% (living more than three years). Figure $1 \mathrm{~A}$ displays the cumulative survival curve from BVZ administration for all 31 recurrent gliomas.

Hemogram pre- and post-bevacizumab treatment determined toxicity: levels of anemia (less than $8 \mathrm{~g} / \mathrm{dL}$ ) appeared in nine patients (29\%), lymphopenia was experienced by $54.8 \%$, and $19 \%$ showed severe lymphopenia (less than 500 lymphocytes $/ \mathrm{mL}$ ). Twenty-nine percent had thrombocytopenia with lower than 50,000 platelets $/ \mathrm{mL}$. Neutropenia appeared in only one patient. Table 2 shows the mean, median, standard deviation, and 95\%CI for levels of neutrophils, lymphocytes, platelets, NLR, and PLR.

\section{Pre-bevacizumab levels of neutrophils or platelets were not related to survival}

The concentrations were considered low if they were below the median, 4950 cells $/ \mathrm{mL}$ for neutrophils and 196,000 cells/mL for platelets. The concentrations were considered high in other cases. We did not find significant differences in overall survival between patients with high and low concentration of neutrophils or platelets. Figure 1B and C shows their survival curves after BVZ treatment with $P$-values of 0.906 and 0.316 for neutrophils and platelets, respectively. 
Table 1. Patients features in diagnosis and progression

\begin{tabular}{|c|c|c|c|c|c|}
\hline & & \multicolumn{2}{|c|}{ 1st diagnosis } & \multicolumn{2}{|c|}{ Progression } \\
\hline & & No. & $\%$ & No. & $\%$ \\
\hline Age (median in years) & & 46 & & 48 & \\
\hline \multirow[t]{3}{*}{ Progression diagnosis } & Radiological & & & 11 & 32.26 \\
\hline & Clinical & & & 1 & \\
\hline & Clinical-radiological & & & 19 & 67.74 \\
\hline \multirow[t]{3}{*}{ KPS } & Median & 90 & & 85 & \\
\hline & KPS $>80$ & 27 & 87.09 & 14 & 45.16 \\
\hline & $\mathrm{KPS} \leq 80$ & 4 & 12.90 & 14 & 45.16 \\
\hline \multirow[t]{7}{*}{ Location } & Frontal & 4 & 12.90 & 4 & 12.90 \\
\hline & Temporal & 4 & 12.90 & 3 & 9.68 \\
\hline & Parietal & 3 & 9.68 & 5 & 16.13 \\
\hline & Occipital & 4 & 12.90 & 5 & 16.13 \\
\hline & Diencephalon & 2 & 6.45 & 1 & 3.22 \\
\hline & Cerebellum & 1 & 3.22 & 1 & 3.22 \\
\hline & Overlapping & 13 & 41.94 & 12 & 38.7 \\
\hline Edema & & 27 & 87.09 & 20 & 67.74 \\
\hline \multirow[t]{4}{*}{ Surgery } & Total & 30 & 96.77 & 6 & 19.35 \\
\hline & Total resection & 15 & 48.39 & 2 & 33.33 \\
\hline & Subtotal resection & 13 & 41.94 & 4 & 66.67 \\
\hline & Biopsy & 2 & 6.45 & 0 & 0 \\
\hline Gliadel & & 10 & 32.26 & 5 & 83.33 \\
\hline \multirow[t]{6}{*}{ Anatomic pathology } & Astrocytoma II & 3 & 9.67 & 2 & 6.66 \\
\hline & Oligodendroglioma II & 1 & 3.23 & 1 & 3.33 \\
\hline & Glioma II & 1 & 3.23 & 1 & 3.33 \\
\hline & Oligodendroglioma III & 2 & 6.45 & 2 & 6.66 \\
\hline & Astrocytoma III & 5 & 16.13 & 5 & 16.66 \\
\hline & GBM and High-grade glioma & 18 & 58.06 & 19 & 63.33 \\
\hline RT & & 29 & 93.55 & 2 & 6.45 \\
\hline Concomitant TMZ & & 25 & 80.64 & 2 & 6.45 \\
\hline \multirow{5}{*}{$\begin{array}{l}\text { Adjuvant } \\
\text { TMZ }\end{array}$} & Total & 23 & 74.19 & 29 & 93.55 \\
\hline & Stupp & 23 & 74.19 & 16 & 55.17 \\
\hline & Cycles (mean) & 7.43 & & 4.38 & \\
\hline & Confidence interval (95\%) & $(5.64-9.23)$ & & $(2.34-6.41)$ & \\
\hline & Metronomic (any time) & 6 & 26 & 13 & 44.83 \\
\hline
\end{tabular}

KPS: karnofsky performance status; No.: number of patients; RT: radiotherapy; TMZ: temozolomide

\section{Patients with high Pre-bevacizumab levels of lymphocytes showed better outcomes}

Patients with levels of lymphocytes above the median $(1260 \mathrm{cell} / \mathrm{s} / \mathrm{mL})$ tended to show better outcomes. This association was close to significance with $P$-value $=0.052$, as shown in Figure $1 \mathrm{D}$. However, both the first $(1019$ cells $/ \mathrm{mL})$ and second tertiles $(1522$ cells $/ \mathrm{mL})$ as cut-off thresholds for lymphocytes showed significant survival differences (with $P$-value $=0.042$ and $P$-value $=0.048$, respectively). Low levels of lymphocytes were associated with worse outcomes, with $P=0.042$ in Figure $1 \mathrm{E}$ and $P=0.048$ in Figure $1 \mathrm{~F}$, choosing the first and second tertiles, respectively.

\section{Pre-bevacizumab NLR or PLR ratios were not individually related to survival}

Patients were classified as negative for NLR if their value was below the median (3.97). Similarly, patients were considered negative for PLR if their value was below the median (186.42). Both negative groups (for NLR or PLR) tended to show better prognoses, with survival curves always above their respective positive groups. Nevertheless, Figure $2 \mathrm{~A}$ and B shows how these differences between positive and negative groups were not significant ( $P$-values of 0.121 for NLR and 0.39 for PLR). The same association was made using tertiles as cut-off thresholds, with similar results. Pre-bevacizumab NLR and PLR ratios were not individually related to survival. 

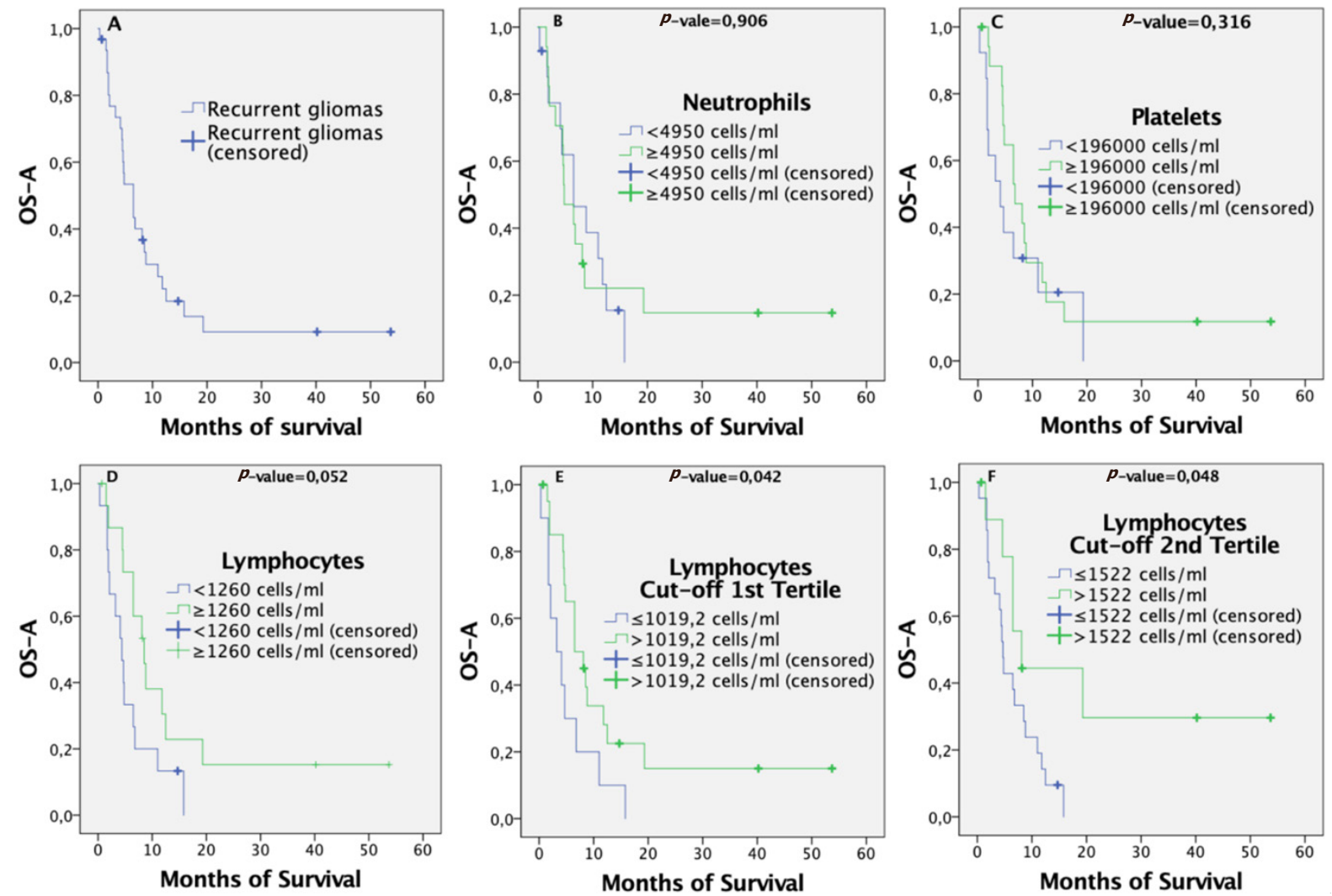

Figure 1. Overall survival from BVZ administration calculated by Kaplan-Meier curves for the total of 31 patients: (A) all patients in the same group; (B) stratified by levels of neutrophils; (C) stratified by levels of platelets; (D-F) stratified by levels of lymphocytes using the median $=1260$ cells $/ \mathrm{mL}$, the first tertile $=1019$ cells $/ \mathrm{mL}$, and the second tertile $=1522 \mathrm{cells} / \mathrm{mL}$ as the cut-offs, respectively. BVZ: bevacizumab

Table 2. Levels of neutrophils, lymphocytes, platelets ( $\mathrm{mg} / \mathrm{dL}), \mathrm{NLR}$, and PLR blood counted before first cycle of bevacizumab

\begin{tabular}{lccclc}
\hline & Neutrophils (mg/dL) & Lymphocytes (mg/dL) & Platelets (mg/dL) & NLR & PLR \\
\hline Mean & 5.67 & 1.26 & 222.03 & 5.71 & 207.19 \\
Median & 4.95 & 1.26 & 196.00 & 3.97 & 186.42 \\
SD & 2.92 & 0.50 & 85.35 & 6.00 & 132.98 \\
$95 \% \mathrm{Cl}$ & $(4.6-6.75)$ & $(1.08-1.45)$ & $(190.73-253.34)$ & $(3.51-7.91)$ & $(158.40-255.97)$ \\
\hline
\end{tabular}

SD: standard deviation; NLR: neutrophil-to-lymphocyte ratio; PLR: platelet-to-lymphocyte ratio

\section{Correlation between inflammation variables}

Lymphocytes were correlated with platelets $(P=0.042)$, NLR $(P=0.025)$, and PLR $(P<0.001)$ only weakly (correlation coefficient $0.368,-0.401$, and -0.589 , respectively). As expected, neutrophils were correlated with NLR $(P>0.001$ and correlation coefficient of 0.676$)$ and platelets with PLR $(P<0.020$ and correlation coefficient of 0.415). Figure $2 \mathrm{C}$ shows that the more NLR increases, the more PLR increases; this association was not statistically significant according to the Spearman test $(P$-value $=0.053 ; 95 \% \mathrm{CI})$.

\section{Combined information PLR-NLR had predictive value}

As association between PLR and NLR was almost significant, we suggest a new patient classification based on both values simultaneously. According to these two variables, patients belong to one of three groups: double positive (both values above their median), double negative (both values below their median), or single positive (the other cases). As the survival of patients with one ratio over the median and the other below it was similar, we classified them into the same group. Median survival was highest for the double- 

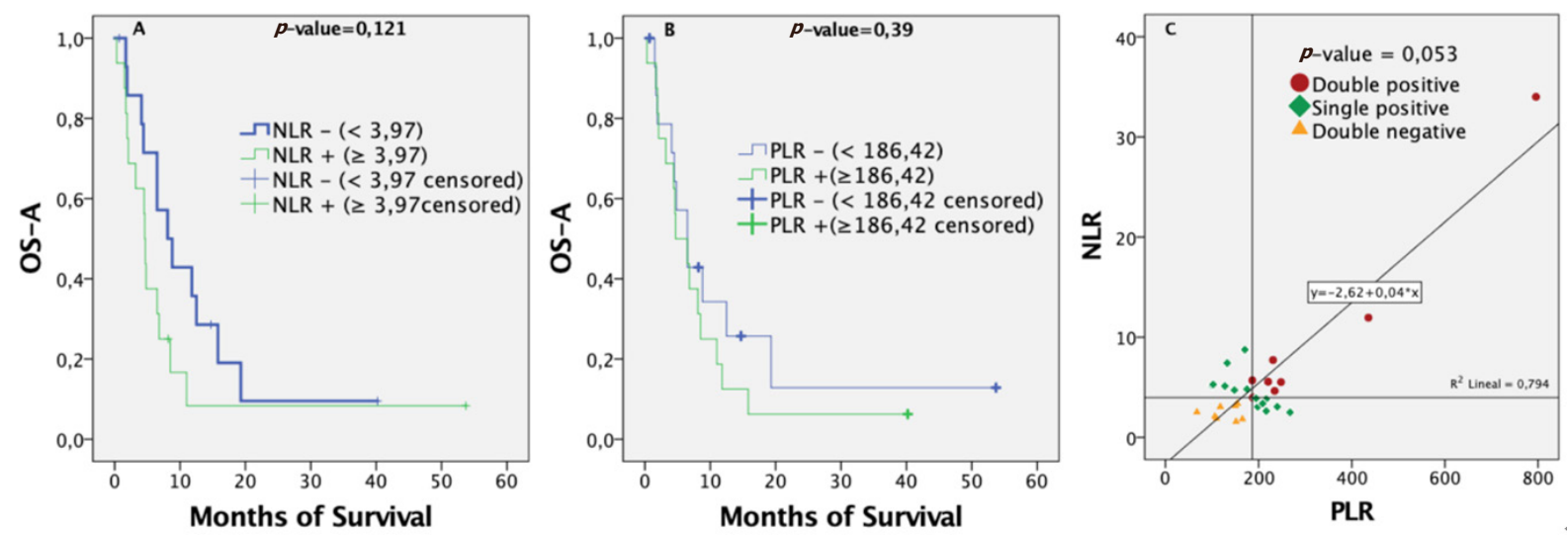

Figure 2. Overall survival from BVZ administration calculated by Kaplan-Meier curves for 31 patients stratified by: (A) NLR positive or negative; (B) PLR positive or negative; (C) Association between NLR and PLR. It seems the more NLR increases, the more PLR increases as well. This association is almost statistically significant following a Spearman test $(P>0.05 ; 95 \% \mathrm{Cl})$. BVZ: bevacizumab; NLR: neutrophil-to-lymphocyte ratio; PLR: platelet-to-lymphocyte ratio

negative group and lowest for the double-positive group. The survival distribution and its median are displayed in Figure 3A. However, statistical difference between the three groups in terms of mean survival was not found using the Kruskal-Wallis test $(P$-value $=0.485 ; 95 \% \mathrm{CI})$. Figure $3 \mathrm{~B}$ displays the survival curves after BVZ treatment for the three groups with $P$-value $=0.125$. However, comparing the doublenegative and single-positive groups, a significant survival difference was observed. The double-positive group had significantly worse prognosis $(P$-value $=0.043,95 \% \mathrm{CI})$ than the non-double-positive group, as shown in Figure 3C.

\section{Similar toxicity for patients classified by combining PLR-NLR information}

The median age was 40.5, 52, and 48 years for the double-negative, single-positive, and double-positive groups, respectively. Although other therapies had been provided previously, Table 3 shows that toxicity was similar in the three groups. RT or TMZ did not influence NLR and PLR values. We focused on neutropenia and thrombocytopenia before BVZ administration and the differences were not significant: $25 \%$ of the double-negative group, $11.1 \%$ of the double-positive group, and $7 \%$ of the single-positive group had neutropenia $P$-value of 0.849 stratifying into double positive $v s$. non-double positive). Moreover, $62 \%$ of the double-negative group had thrombocytopenia, whereas $22 \%$ and $28.6 \%$ appeared in the double-positive and single-positive groups, respectively ( $P$-value of 0.324 stratifying into two groups). Neither NLR nor PLR was related to hematologic BVZ toxicity: $22 \%$ and $21 \%$ of double-positive group and single-positive group had neutropenia and not a single case of double-negative had it ( $P$-value of 0.555 stratifying into two groups). Fifty percent of double-negative group, $44 \%$ of double-positive group, and $28.6 \%$ of single-positive group developed thrombocytopenia ( $P$-value 0.675 stratifying into two groups).

\section{Lymphocyte level was the best independent predictor}

Lymphocyte level before BVZ administration was the best independent predictor of overall survival, as displayed in Table 4 ( $\mathrm{HR}=0.34 ; 95 \% \mathrm{CI}: 0.145-0.81 ; P=0.015)$. The area under the ROC curve was 0.823 and the optimal cut-off value was $1645 \mathrm{cell} / \mathrm{mL}$ ( 0.80 sensitivity and 0.85 specificity). Interestingly, the number of BVZ cycles was not related to lymphopenia, and differences in the distribution of age, sex, KPS, extent of resection, alcohol/tobacco use, and histological diagnoses were not significant.

\section{Lymphocyte level and outcome depending on tumor localization}

Figure 4 shows seven different localizations assessed using MRI: frontal (four patients), temporal (four patients), parietal (three patients), cerebellar (one patient), occipital (four patients), overlapping (thirteen 

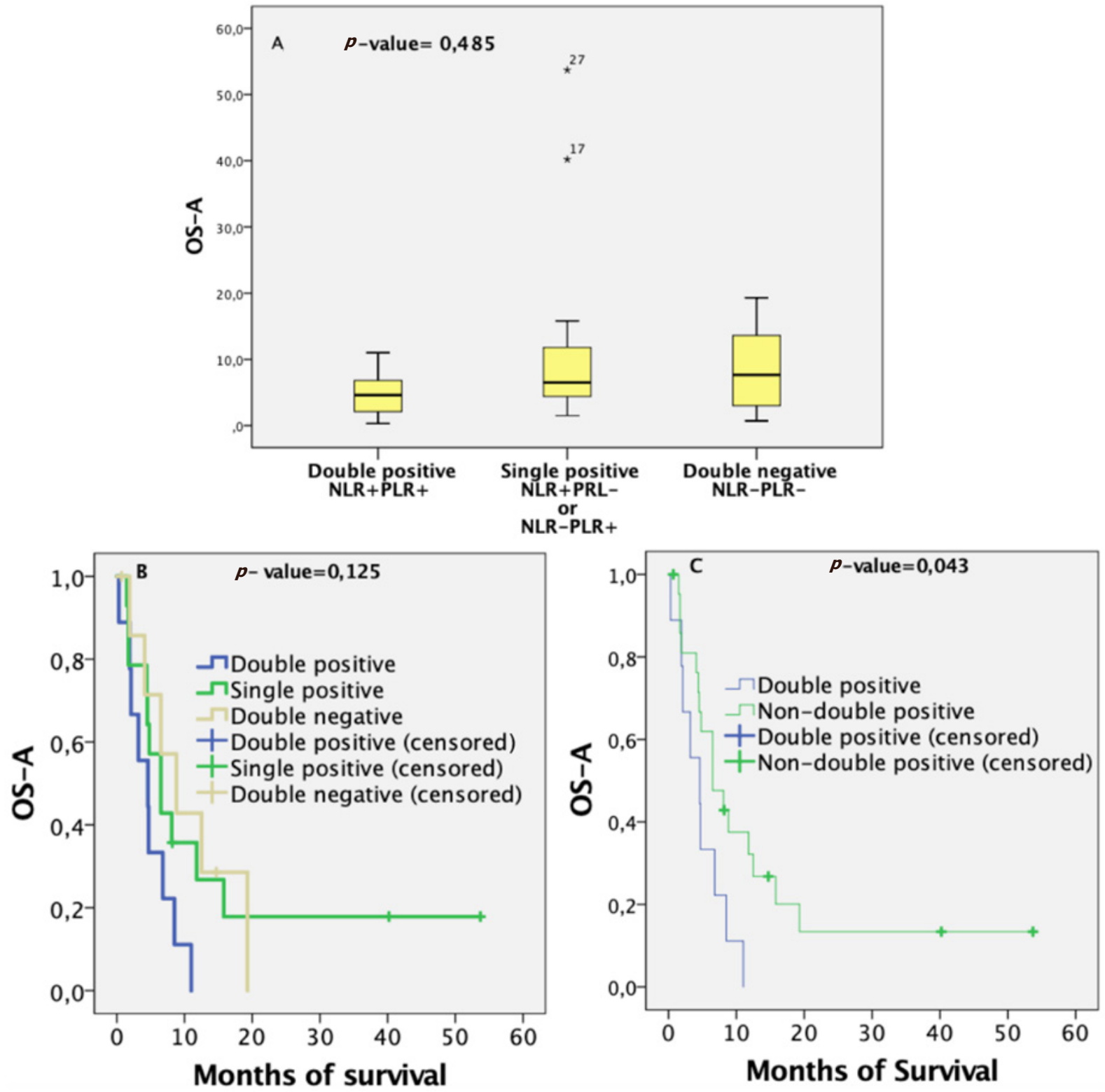

Figure 3. Predictive survival ability combining PLR-NLR information: (A) diagram boxes comparing median survival after BVZ administration among the three groups; (B) overall survival from BVZ administration calculated by Kaplan-Meier curves for 31 patients stratified into three groups, namely double positive, double negative, and single positive; (C) overall survival from BVZ administration calculated by Kaplan-Meier curves for 31 patients stratified into a double-positive group and otherwise. BVZ: bevacizumab; NLR: neutrophil-to-lymphocyte ratio; PLR: platelet-to-lymphocyte ratio

patients), and diencephalon (two patients). Figure 4A exhibits the distribution of pretreatment lymphocyte levels depending on tumor localization. Patients with frontal or temporal tumors tended to show lower lymphocyte values. Figure $4 \mathrm{~B}$ shows the OS after BVZ treatment for the same groups of patients. The three patients with the best outcome were found in the overlapping group. The parietal group had a high lymphocyte level but also a high OS with a small standard deviation in both cases. No statistical differences were found between patients with tumors in the left and right hemispheres, in terms of either lymphocyte levels or survival.

\section{Limitations}

The main limitations of our study are its retrospective design and the small and diverse sample. 
Table 3. Clinical characteristics of patients according to PLR-NLR groups

\begin{tabular}{llllll}
\hline & \multicolumn{1}{c}{$\begin{array}{c}\text { Double Positive } \\
(\boldsymbol{n}=\mathbf{9})\end{array}$} & $\begin{array}{c}\text { Single Positive } \\
(\boldsymbol{n}=\mathbf{1 4})\end{array}$ & $\begin{array}{c}\text { Double Negative } \\
(\boldsymbol{n}=\mathbf{8})\end{array}$ & $\begin{array}{c}\boldsymbol{P} \text {-value } \\
(\mathbf{3} \text { groups) }\end{array}$ & $\begin{array}{c}\boldsymbol{P} \text {-value } \\
(\mathbf{2} \text { groups) }\end{array}$ \\
\hline Pre-BVZ toxicity & $(\mathrm{Yes} / \mathrm{No})$ & $(\mathrm{Yes} / \mathrm{No})$ & $($ Yes/No) & 0.984 & 0.873 \\
Anemia & $3 / 6$ & $5 / 9$ & $3 / 5$ & & 0.849 \\
Neutropenia & $1 / 8(11.1 \% / 88.9 \%)$ & $1 / 13(7 \% / 93 \%)$ & $2 / 6(25 \% / 75 \%)$ & 0.477 & 0.129 \\
Lymphopenia & $5 / 4$ & $11 / 3$ & $7 / 1$ & 0.285 & 0.627 \\
Leukopenia & $1 / 8$ & $2 / 12$ & $2 / 6$ & 0.716 & 0.324 \\
Thrombocytopenia & $2 / 7(22 \% / 78 \%)$ & $4 / 10(28.6 \% / 71.4 \%)$ & $5 / 3(62.5 \% / 37.5)$ & 0.171 & 0.675 \\
Post-BVZ toxicity & $(Y e s / \mathrm{No})$ & $(\mathrm{Yes} / \mathrm{No})$ & $(\mathrm{Yes} / \mathrm{No})$ & 0.203 & \\
Anemia & $4 / 5$ & $7 / 7$ & $1 / 7$ & & 0.555 \\
Neutropenia & $2 / 7(22 \% / 78 \%)$ & $3 / 11(21 \% / 78.5 \%)$ & $0 / 8(0 \% / 100 \%)$ & 0.354 & 0.593 \\
Lymphopenia & $7 / 2$ & $10 / 4$ & $5 / 3$ & 0.786 & 0.499 \\
Leukopenia & $1 / 8$ & $1 / 13$ & $0 / 8$ & 0.642 & 0.675 \\
Thrombocytopenia & $4 / 5(44 \% / 56 \%)$ & $4 / 10(28.6 \% / 71.4 \%)$ & $4 / 4(50 \% / 50 \%)$ & 0.560 & \\
\hline
\end{tabular}

NLR: neutrophil-to-lymphocyte ratio; PLR: platelet-to-lymphocyte ratio; BVZ: bevacizumab; 3 groups: double positive, single positive, or double negative; 2 groups: double positive $v s$. non-double positive

Table 4. Uni- and multivariable analysis (Cox regression) for the most representative variables associated with OS after BVZ treatment

\begin{tabular}{|c|c|c|c|c|}
\hline \multirow{2}{*}{ Variables } & \multicolumn{2}{|c|}{ OS-A (univariable) } & \multicolumn{2}{|c|}{ OS-A (multivariable) } \\
\hline & $\mathrm{HR},(95 \% \mathrm{Cl})$ & $P$-value & $\mathrm{HR},(95 \% \mathrm{Cl})$ & $P$-value \\
\hline Age as continuous variable & $1.02(0.96-1.05)$ & 0.306 & & \\
\hline Gender & $1.25(0.52-2.98)$ & 0.619 & & \\
\hline Neutrophil Level (cells/mL) & $1.03(0.89-1.19)$ & 0.704 & & \\
\hline Median: < 4950 vs. $\geq 4950$ & $0.95(0.43-2.11)$ & 0.906 & & \\
\hline Platelet Level (cells/mL) & $1.00(0.99-1.00)$ & 0.145 & & \\
\hline Median: <196,000 vs. $\geq 196,000$ & $0.33(0.68-3.23)$ & 0.316 & & \\
\hline Lymphocyte Level (cells/mL) & $0.34(0.145-0.81)$ & $0.015^{\star}$ & & \\
\hline Median: $<1260$ vs. $\geq 1260$ & $2.15(0.97-4.80)$ & 0.052 & & \\
\hline First tertile: $<1019$ vs. $\geq 1019$ & $2.25(1.00-5.04)$ & $0.042^{\star}$ & & \\
\hline Second tertile: $<1522$ vs. $\geq 1522$ & $2.62(0.96-7.10)$ & $0.048^{*}$ & & \\
\hline NLR & $1.04(0.99-1.10)$ & 0.105 & & \\
\hline Median: (<3.97vs. $\geq 3.97)$ & $0.54(0.24-1.20)$ & 0.121 & & \\
\hline PLR & $1.00(1.00-1.00)$ & 0.249 & & \\
\hline Median: (<186.42 vs. $\geq 186.42)$ & $0.71(0.32-1.56)$ & 0.390 & & \\
\hline PLR-NLR (Double positive vs. non-double positive) & $2.35(0.99-5.56)$ & $0.043^{\star}$ & & \\
\hline Neutropenia & $0.93(0.32-2.72)$ & 0.890 & & \\
\hline Lymphopenia & $2.23(0.82-6.10)$ & 0.118 & & \\
\hline Leukopenia & $0.77(20.29-2.06)$ & 0.604 & & \\
\hline Thrombocytopenia & $1.20(0.52-2.73)$ & 0.682 & & \\
\hline Anemia & $2.22(0.92-5.33)$ & 0.076 & & \\
\hline Lymphocyte Level (cells/mL) & & & $0.42(0.16-1.10)$ & $0.078^{\star}$ \\
\hline PLR-NLR (Double positive vs. non-double positive) & & & $1.50(0.55-4.04)$ & 0.436 \\
\hline
\end{tabular}

${ }^{\star} P$-values significant. OS: overall survival; BVZ: bevacizumab; OS-A: overall survival from bevacizumab administration; HR: hazard ratio; NLR: neutrophil-to-lymphocyte ratio; PLR: platelet-to-lymphocyte ratio

\section{DISCUSSION}

Low-grade gliomas may undergo aggressive transformation, becoming high-grade gliomas, and they also tend to recur after a few months ${ }^{[37]}$. It is still unclear what the best therapeutic option for those recurrent glioma patients who have already received the standard radio- and chemotherapy actually is. Scientists are trying to find: (1) new treatments to prolong OS and quality of life; and (2) biomarkers that predict the response to these treatments. The FDA approved BVZ as a single agent for treatment of recurrent GBM with poor or refractory response to other therapies ${ }^{[17]}$. The European Medicines Agency rejected this indication, although some studies have questioned the decision ${ }^{[18]}$. No new agents have been approved 

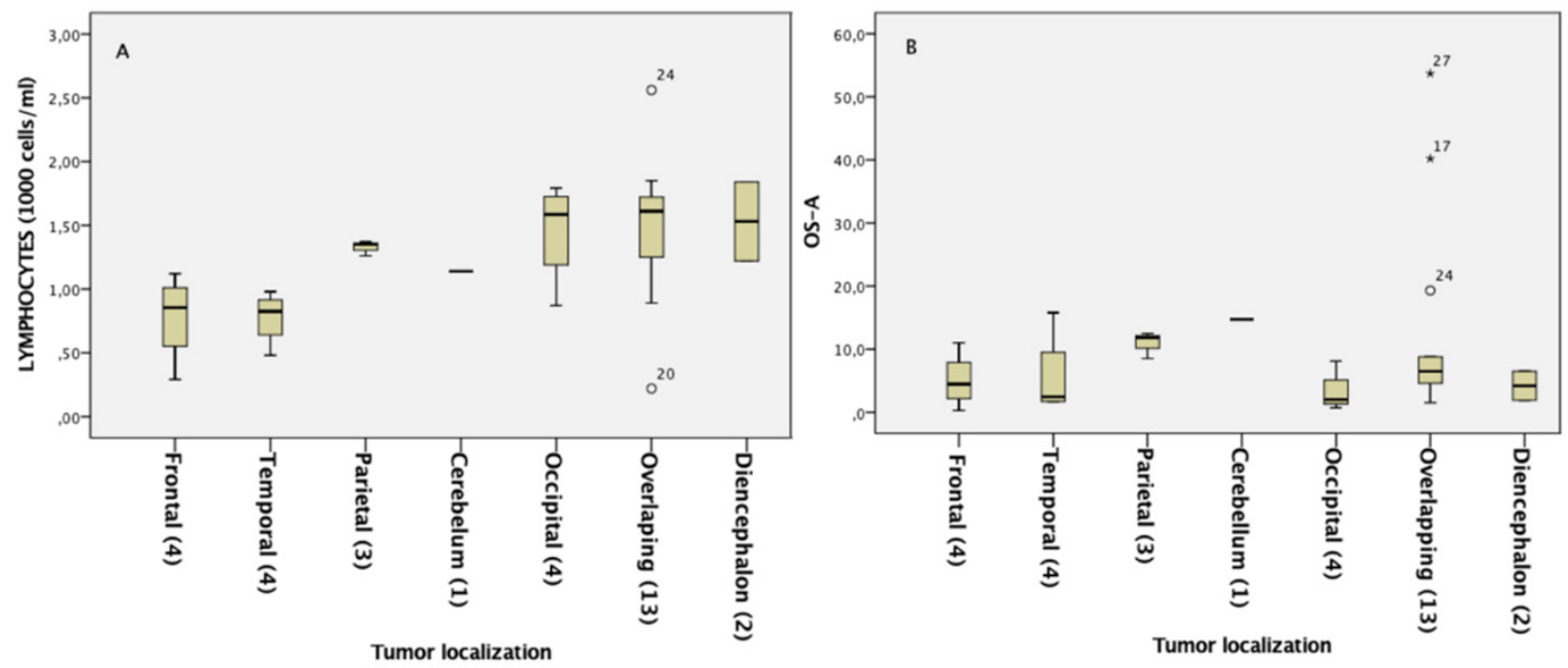

Figure 4. Lymphocyte levels and outcomes with patients classified by tumor localization: (A) lymphocyte level diagram box depending on tumor localization; (B) diagram box of overall survival from BVZ administration depending on tumor localization. BVZ: bevacizumab

for second-line therapy of GBM in Europe since the approval of TMZ in 1999. It has been reported that BVZ enhances the effect of RT and TMZ, allowing chemotherapy and oxygen to better perfuse within the tumor $^{[14]}$. BVZ can attenuate tumor-associated brain edema and thus improve patient symptoms due to a reduction in steroid use ${ }^{[10]}$. Nevertheless, it seems that only some patients benefit; thus, it would be useful to find biomarkers that determine those who might respond beforehand ${ }^{[18]}$.

Inflammation and cancer are linked: high levels of neutrophils and platelets are related to poor prognosis for several cancers while high lymphocytic infiltration is associated with improved survival and superior response to systemic therapy ${ }^{[25,28]}$. However, there are few studies focused on prognostic inflammatory biomarkers for recurrent gliomas ${ }^{[30-35]}$ and even fewer focused on BVZ response after recurrence.

To our knowledge, no one has analyzed the pretreatment levels of inflammatory indices with survival in recurrent gliomas treated with BVZ. We analyzed the correlation between survival and inflammatory components measured before BVZ administration in 31 recurrent gliomas: neutrophils, lymphocytes, platelet counts, the ratios of NLR and PLR, and the combination PLR-NLR.

Our results suggest that pre-BVZ levels of neutrophils and platelets do not seem to act as biomarkers. However, the group of patients with high levels of lymphocytes lived longer than the others. This result is robust since several cut-off values showed significant differences between groups. We recommend using a threshold for lymphocytes between 1019 cells $/ \mathrm{mL}$ (first tertile) and 1522 cells $/ \mathrm{mL}$ (third tertile). Then, we do not know if lymphocyte infiltration before BVZ treatment could improve overall survival for recurrent glioma patients.

Since lymphocytes were the most powerful biomarker, we analyzed if it was related to the location of the tumor. Those patients with frontal or temporal tumors tended to have lower lymphocyte values. The three patients with the best outcome were found in the overlapping group. The parietal group had a high lymphocyte level but also a high OS with small standard deviation in both cases. Unfortunately, the low number of individuals per group did not allow us to determine differences between groups. However, these preliminary results could inspire other groups and motivate a deeper study.

The relation between survival and pretreatment NLR or PLR was not statistically significant and survival curves where similar $(P$-value $=0.121$ and $P$-value $=0.39$, respectively $)$. The Spearman correlation $\mathrm{p}$-value 
between NLR and PLR was 0.053 . We would expect to have a more significant correlation value with a larger number of patients. It is noteworthy that the survival curve for NLR-PLR positive patients was below the survival curve of NLR-PLR negative patients. This result fits very well with previous results that a NLR $>4$ prior to second surgery was a poor prognostic factor in GBM, and later progression was associated with longer survival in patients but not in longer survival after second surgery ${ }^{[32]}$.

The combined inflammatory information PLR-NLR may affect the evolution of high-grade gliomas at recurrence treated with bevacizumab. In fact, the double-positive category is associated with the worst prognosis $(P$-value $=0.043)$. This merger had not been carried out in the studies that were previously reviewed. We hypothesize that patients with double-positive PLR-NLR have more angiogenesis and the BVZ effect is smaller, having worse prognosis. Nevertheless, a deeper study would be necessary.

Regarding toxicity, we found fewer patients suffering lymphopenia and leukopenia with treatment at recurrence than at diagnosis. However, more patients had anemia, neutropenia, or thrombocytopenia at recurrence. Some of them also received other therapies after progression such as second surgery or TMZ. Although 55.17\% of patients received Stupp treatment after progression (4.5 average cycles), later neutropenia was not affected. A complication when studying recurrence is the possible modification of our results due to previous administration of therapies or development of infections. In principle, the toxicity the patients experience in prior therapy could influence posterior NLR and PLR levels. It is believed that patients with more toxicity respond better to therapy. In our study, toxicity was similar in all groups: neither NLR nor PLR was related to hematological BVZ toxicity.

This study classified recurrent glioma patients who receive more benefit from the treatment with BVZ considering new immune markers: both lymphocyte levels and the combination of PLR-NLR could be employed as non-invasive hematological prognostic markers for recurrent high-grade gliomas treated with BVZ. However, lymphocyte level before BVZ administration was the best independent predictor of overall survival from bevacizumab administration (OS-A). A close relationship appeared between inflammation and angiogenesis, although this is not very well understood yet.

\section{DECLARATIONS}

\section{Acknowledgments}

We are most grateful to the Hospital Universitario de Gran Canaria Doctor Negrin Facility of Las Palmas de Gran Canaria and especially to the Radio Oncology Service for their technical support.

\section{Authors' contributions}

Wrote the paper, edited figures, developed statistical analysis and discussed results: Martínez-González A Collected data and designed the protocol: Cabrera R

Designed the study and protocol: Lloret $\mathrm{M}$

Designed the study and protocol, discussed results and revised the manuscript: Lara PC All authors approved the final version of the manuscript.

\section{Availability of data and materials}

Not applicable.

\section{Financial support and sponsorship}

This work has been supported by Ministerio de Economía y Competitividad/FEDER, Spain [grant number MTM2015-71200-R] and James S. Mc. Donnell Foundation 21st Century Science Initiative in Mathematical and Complex Systems Approaches for Brain Cancer [Special Initiative Collaborative, Planning Grant 220020420 and Collaborative award 220020450]. 


\section{Conflicts of interest}

All authors declared that there are no conflicts of interest.

\section{Ethical approval and consent to participate}

This study was carried out in accordance to the protocol "PATRORA" approved by the Agencia Española de Medicamentos y Productos Sanitarios (ref: PLJ-BEV-2016-01) and by the Hospital Universitario de Gran Canaria Doctor Negrin committee (ref: 170007).

\section{Consent for publication}

Not applicable.

\section{Copyright}

(c) The Author(s) 2020.

\section{REFERENCES}

1. Barciszewska AM, Gurda D, Głodowicz P, Nowak S, Naskręt-Barciszewska MZ. A new epigenetic mechanism of temozolomide action in glioma cells. PLoS One 2015;10:e0136669.

2. Stupp R, Dietrich PY, Ostermann Kraljevic S, Pica A, Maillard I, et al. Promising survival for patients with newly diagnosed glioblastoma multiforme treated with concomitant radiation plus temozolomide followed by adjuvant temozolomide. J Clin Oncol 2002;20:1375-82.

3. Jalbert LE, Neill E, Phillips JJ, Lupo JM, Olson MP, et al. Magnetic resonance analysis of malignant transformation in recurrent glioma. Neuro Oncol 2016;18:1169-79.

4. Rempel SA, Dudas S, Ge S, Gutiérrez JA. Identification and localization of the cytokine SDF1 and its receptor, CXC chemokine receptor 4, to regions of necrosis and angiogenesis in human glioblastoma. Clin Cancer Res 2000;6:102-11.

5. Brada M, Hoang-Xuan K, Rampling R, Dietrich PY, Dirix LY, et al. Multicenter phase II trial of temozolomide in patients with glioblastoma multiforme at first relapse. Ann Oncol 2001;12:259-66.

6. Peak SJ, Levin VA. Role of bevacizumab therapy in the management of glioblastoma. Cancer Manag Res 2010;2:97-104.

7. Shao R, Taylor SL, Oh DS, Schwartz LM. Vascular heterogeneity and targeting: the role of YKL-40 in glioblastoma vascularization. Oncotarget 2015;6:40507-18.

8. Gil-Gil MJ, Mesia C, Rey M, Bruna J. Bevacizumab for the treatment of glioblastoma. Clin Med Insights Oncol 2013;7:123-35.

9. Nagane M. Dose-dense temozolomide: is it still promising?. Neurol Med Chir (Tokyo) 2015;55:38-49.

10. Xu H, Rahimpour S, Nesvick CL, Zhang X, Ma JY, et al. Activation of hypoxia signaling induces phenotypic transformation of glioma cells: implications for bevacizumab antiangiogenic therapy. Oncotarget 2015;6:11882-93.

11. Martínez-González A, Calvo GF, Pérez Romasanta LA, Pérez-García VM. Hypoxic cell waves around necrotic cores in glioblastoma: a biomathematical model and its therapeutic implications. Bull Math Biol 2012;74:2875-96.

12. Ke Q, Costa M. Hypoxia-inducible factor-1 (HIF-1). Mol Pharmacol 2006;70:1469-80.

13. Friedman HS, Prados MD, Wen PY, Mikkelsen T, Schiff D, et al. Bevacizumab alone and in combination with irinotecan in recurrent glioblastoma. J Clin Oncol 2009;27:4733-40.

14. Koukourakis GV, Kouloulias V, Zacharias G, Papadimitriou C, Pantelakos P, et al. Temozolomide with radiation therapy in high grade brain gliomas: pharmaceuticals considerations and efficacy; a review article. Molecules 2009;14:1561-77.

15. Takano S, Ishikawa E, Nakai K, Matsuda M, Masumoto T, et al. Bevacizumab in Japanese patients with malignant glioma: from basic research to clinical trial. Onco Targets Ther 2014;7:1551-62.

16. Poulsen HS, Urup T, Michaelsen SR, Staberg M, Villingshøj M, Lassen U. The impact of bevacizumab treatment on survival and quality of life in newly diagnosed glioblastoma patients. Cancer Manag Res 2014;6:373-87.

17. Gatson NN, Chiocca EA, Kaur B. Anti-angiogenic gene therapy in the treatment of malignant gliomas. Neurosci Lett 2012;527:62-70.

18. Balañá C, Etxaniz O, Bugés C, Martínez A. Approval denied by the European Medicines Agency (EMA) for bevacizumab in the treatment of high-grade glioma recurrence: a good idea or a grave error?. Clin Transl Oncol 2011;13:209-10.

19. Stupp R, Mason WP, van den Bent MJ, Weller M, Fisher B, et al. Radiotherapy plus concomitant and adjuvant temozolomide for glioblastoma. N Engl J Med 2005;352:987-96.

20. Pérez-Beteta J, Martínez-González A, Molina D, Amo-Salas M, Luque B, et al. Glioblastoma: does the pre-treatment geometry matter? A postcontrast T1 MRI-based study. Eur Radiol 2017;27:1096-104.

21. Molina D, Pérez-Beteta J, Luque B, Arregui E, Calvo M, et al. Tumour heterogeneity in glioblastoma assessed by MRI texture analysis: a potential marker of survival. Br J Radiol 2016;89:20160242.

22. Molina D, Pérez-Beteta J, Martínez-González A, Sepúlveda JM, Peralta S, et al. Geometrical measures obtained from pretreatment postcontrast T1 weighted MRIs predict survival benefits from bevacizumab in glioblastoma patients. PLoS One 2016;11:e0161484.

23. Manneh Kopp RA, Sepúlveda-Sánchez JM, Ruano Y, Toldos O, Pérez Núñez A, et al. Correlation of radiological and immunochemical parameters with clinical outcome in patients with recurrent glioblastoma treated with Bevacizumab. Clin Transl Oncol 2019;21:1413-23.

24. Que Y, Qiu H, Li Y, Chen Y, Xiao W, et al. Preoperative platelet-lymphocyte ratio is superior to neutrophil-lymphocyte ratio as a 
prognostic factor for soft-tissue sarcoma. BMC Cancer 2015;15:648.

25. Feng Z, Wen H, Bi R, Ju X, Chen X, et al. Preoperative neutrophil-to-lymphocyte ratio as a predictive and prognostic factor for highgrade serous ovarian cancer. PLoS One 2016;11:e0156101.

26. Zhou X, Du Y, Huang Z, Xu J, Qiu T, et al. Prognostic value of PLR in various cancers: a meta-analysis. PLoS One 2014;9:e101119.

27. D'Emic N, Engelman A, Molitoris J, Hanlon A, Sharma NK, et al. Prognostic significance of neutrophil-lymphocyte ratio and plateletlymphocyte ratio in patients treated with selective internal radiation therapy. J Gastrointest Oncol 2016;7:269-77.

28. Templeton AJ, Ace O, McNamara MG, Al-Mubarak M, Vera-Badillo FE, et al. Prognostic role of platelet to lymphocyte ratio in solid tumors: a systematic review and meta-analysis. Cancer Epidemiol Biomarkers Prev 2014;23:1204-12.

29. Passardi A, Scarpi E, Cavanna L, Dall'Agata M, Tassinari D, et al. Inflammatory indexes as predictors of prognosis and bevacizumab efficacy in patients with metastatic colorectal cancer. Oncotarget 2016;7:33210-9.

30. Templeton AJ, McNamara MG, Šeruga B, Vera-Badillo FE, Aneja P, et al. Prognostic role of neutrophil-to-lymphocyte ratio in solid tumors: a systematic review and meta-analysis. J Natl Cancer Inst 2014;106:dju124.

31. Alexiou GA, Vartholomatos E, Voulgaris S. Prognostic value of neutrophil-to-lymphocyte ratio in patients with glioblastoma. J Neurooncol 2013;115:521-2.

32. McNamara MG, Lwin Z, Jiang H, Templeton AJ, Zadeh G, et al. Factors impacting survival following second surgery in patients with glioblastoma in the temozolomide treatment era, incorporating neutrophil/lymphocyte ratio and time to first progression. J Neurooncol 2014;117:147-52.

33. Han S, Liu Y, Li Q, Li Z, Hou H, et al. Pre-treatment neutrophil-to-lymphocyte ratio is associated with neutrophil and T-cell infiltration and predicts clinical outcome in patients with glioblastoma. BMC Cancer 2015;15:617.

34. Audureau E, Chivet A, Ursu R, Corns R, Metellus P, et al. Prognostic factors for survival in adult patients with recurrent glioblastoma: a decision-tree-based model. J Neurooncol 2018;136:565-76.

35. Bertaut A, Truntzer C, Madkouri R, Kaderbhai CG, Derangère V, et al. Blood baseline neutrophil count predicts bevacizumab efficacy in glioblastoma. Oncotarget 2016;7:70948-58.

36. Vaios EJ, Winter SF, Muzikansky A, Nahed BV, Dietrich J. Eosinophil and lymphocyte counts predict bevacizumab response and survival in recurrent glioblastoma. Neuro-Oncology Advances 2020;2:vdaa031.

37. Bogdanska MU, Bodnar M, Piotrowska MJ, Murek M, Schucht P, et al. A mathematical model describes the malignant transformation of low grade gliomas: Prognostic implications. PLoS One 2017;12:e0179999. 